\title{
A Path Analysis of Latino Parental, Teenager and Cultural Variables in Teenagers' Sexual Attitudes, Norms, Self-Efficacy, and Sexual Intentions ${ }^{1}$
}

\author{
Vanessa Pirani Gaioso² \\ Antonia Maria Villarruel ${ }^{3}$ \\ Lynda Anne Wilson ${ }^{4}$ \\ Andres Azuero ${ }^{5}$ \\ Gwendolyn Denice Childs ${ }^{6}$ \\ Susan Lane Davies ${ }^{7}$
}

Objective: to test a theoretical model based on the Parent-Based Expansion of the Theory of Planned Behavior examining relation between selected parental, teenager and cultural variables and Latino teenagers' intentions to engage in sexual behavior. Method: a cross-sectional correlational design based on a secondary data analysis of 130 Latino parent and teenager dyads. Results: regression and path analysis procedures were used to test seven hypotheses and the results demonstrated partial support for the model. Parent familism and knowledge about sex were significantly associated with parents' attitudes toward sexual communication with their teenagers. Parent Latino acculturation was negatively associated with parents' self-efficacy toward sexual communication with their teenagers and positevely associated with parents' subjective norms toward sexual communication with their teenagers. Teenager knowledge about sex was significantly associated with higher levels of teenagers' attitudes and subjective norms about sexual communication with parents. Only the predictor of teenagers' attitudes toward having sex in the next 3 months was significantly associated with teenagers' intentions to have sex in the next 3 months. Conclusion: the results of this study provide important information to guide future research that can inform development of interventions to prevent risky teenager sexual behavior among Latinos.

Descriptors: Adolescent Behavior; Hispanic Americans; Sexual Behavior; Parent-Child Relations; Communication; Culture.

1 Supported by National Institutes of Health Grant, United States, process \# 5R21NR010457-02.

$2 \mathrm{PhD}$, Instructor, University of Alabama at Birmingham, School of Nursing, Birmingham, AL, United States.

3 PhD, Full Professor, University of Pennsylvania, School of Nursing, Philadelphia, PA, United States.

${ }^{4} \mathrm{PhD}$, Full Professor, University of Alabama at Birmingham, School of Nursing, Birmingham, AL, United States.

5 PhD, Associate Professor, University of Alabama at Birmingham, School of Nursing, Birmingham, AL, United States.

${ }^{6} \mathrm{PhD}$, Assistant Professor, University of Alabama at Birmingham, School of Nursing, Birmingham, AL, United States.

$7 \mathrm{PhD}$, Associate Professor, University of Alabama at Birmingham, School of Public Health, Birmingham, AL, United States.

Corresponding Author: Vanessa Pirani Gaioso University of Alabama at Birmingham. School of Nursing 1714 9th Avenue South, suite LRC155

35294-1270, Birmingham, Alabama, United States

E-mail: vgaioso@uab.edu
Copyright ( 2015 Revista Latino-Americana de Enfermagem This is an Open Access article distributed under the terms of the Creative Commons Attribution Non-Commercial License (CC BY-NC).

This license lets others distribute, remix, tweak, and build upon your work non-commercially, and although their new works must also acknowledge you and be non-commercial, they don't have to license their derivative works on the same terms. 


\section{Introduction}

Over the past two decades, the number of Latinos has grown rapidly in the United States (U.S.), and Latinos now comprise the nation's largest minority ethnic group at $17 \%$ of the total population. The U.S. Latino population is young, with a median age of 27 years $^{(1)}$. Latino teenagers are more likely than white teenagers to have had more than four sexual partners during their lives, to initiate sexual activity before the age of 13 and to evade using condoms or birth control methods (2). As a result, Latino teenagers suffer from disproportionally higher rates of sexually transmitted diseases, and in 2011 accounted for $21 \%$ of diagnoses of HIV infection(3).

Findings from numerous studies on minority teenagers have supported the view of complex influences at multiple levels of the social environment on Latino teenager sexual behavior. These influences include family, peer groups, schools and neighborhoods, and race or ethnicity ${ }^{(4)}$. Three potentially important external influences are the parents' levels of acculturation(5), perceived value of familism(6), and levels of knowledge about pregnancy and sexually transmitted diseases (STDs) ${ }^{(7)}$. Latino acculturation means the extent to which the individual is oriented to Latino customs or the culture of the new country. Familism values are key components of Latino culture and refer to an individual's perceived value of family unity, closeness, and interconnectedness between the nuclear family and extended family members ${ }^{(8)}$. However, the quantity and quality of parentteenager sexual communication has been suggested as the best predictor on teenager risky sexual behavior(9).

The purpose of this study was to test a theoretical framework based on the Parent Expansion of the Theory of Planned Behavior (PETPB) model examining relationships between selected parental, teenager and cultural variables and Latino teenagers' intentions to engage in sexual behavior. The PETPB model ${ }^{(10)}$ is an ecological expansion of the Theory of Planned Behavior which incorporates the influences of parents' behavior on teenager risky sexual behavior. The model proposes that HIV-risk related sexual behavior would be determined by the teenager's intentions to engage in risky sexual behavior. The model further proposes that teenager's intentions to engage in risky sexual behavior would be determined by his/her behavioral beliefs, normative beliefs, and control beliefs. In addition, the PETPB model proposes that parental behavioral, normative, and control beliefs are influenced by external factors and subsequently influence parents' intentions to talk with their teenagers about sex which is viewed as an external influence on the teenager.

Figure 1 elucidates the adaptation of the PETPB model and the various path analyses and hypotheses that were tested in the proposed study.

Paths $a, b$, and $c$ (Hypothesis 1 ) in the model suggest that parents who have higher levels of Latino acculturation,

External influences on Parent:

External influences on Adolescent:

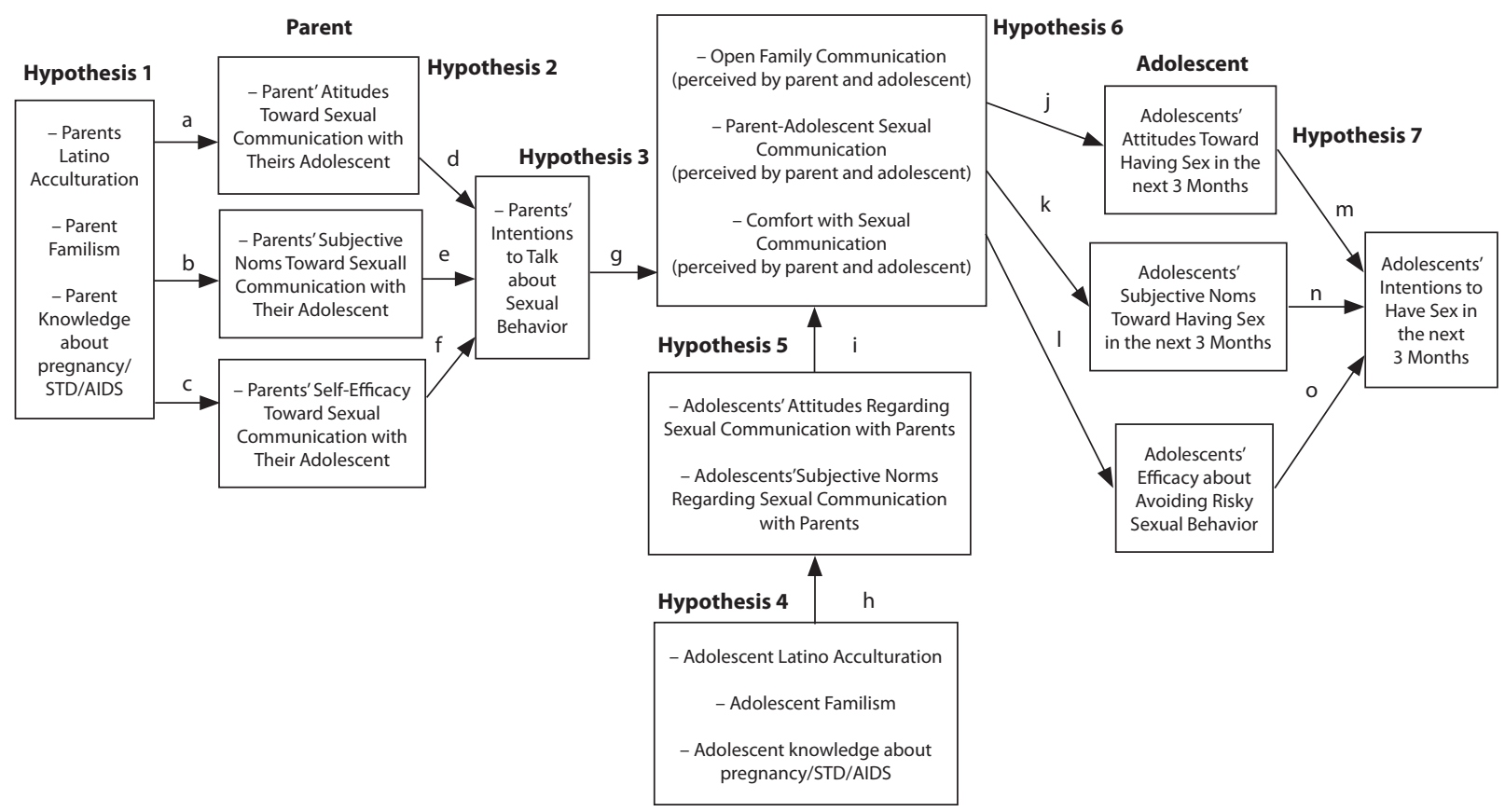

Note: All paths assumed positive association (direct relationships); except the paths $\mathrm{j}, \mathrm{k}$ and o that are inverse relationships.

Figure 1 - Conceptual Model Used in the Present Study 
familism values, and knowledge about pregnancy/STD/ AIDS would have more favorable attitudes, subjective norms, and self-efficacy toward sexual communication with their teenager. Other researchers have found positive relationships between parent-teenager communication and parental level of Latino acculturation ${ }^{(5)}$ and knowledge about sexual topics(7), as well as parental level of familism and parents' self-efficacy toward parent-teenager sexual communication ${ }^{(6)}$.

Paths $d, e$, and $f$ (Hypothesis 2) suggest that more positive parental attitudes, greater subjective norms and higher levels of self-efficacy toward sexual communication with their teenager are associated with higher levels of parents' intentions to talk about sexual behavior with their teenagers. Previous studies have demonstrated positive relationships between parent-teenager sexual communication and parents' attitudes, subjective norms, and self-efficacy toward sexual communication(6,11-14).

Path $g$ (Hypothesis 3) suggests that higher levels of parental intentions to talk about sexual behavior with their teenagers are associated with greater open family communication, parent-teenager sexual communication, and parent-teenager comfort with sexual communication, reported by both parents and teenagers.

Path $h$ (Hypothesis 4) suggests that higher teenager levels of Latino acculturation, familism values, and knowledge about pregnancy/STD/AIDS are associated with higher levels of teenagers' attitudes and subjective norms about sexual communication with parents. Other researchers have reported positive associations between teenager levels of parent-teenager sexual communication and levels of acculturation ${ }^{(15)}$, familism values $^{(6)}$ and knowledge about sex ${ }^{(16)}$.

Path $i$ (Hypothesis 5) suggests that higher levels of teenagers' attitudes and subjective norms regarding sexual communication with parents are associated with higher levels of parents' and teenagers' perceptions of open family communication, parent-teenager sexual communication, and comfort with sexual communication. Findings from one study (that did not include Latino parents and teenagers) suggested a positive association between measures of teenagers' beliefs and subjective norms toward parent-teenager sexual communication and the amount of parent-teenager communication about sexuality reported only by the teenagers ${ }^{(17)}$.

Paths $j, k$ and $/$ (Hypothesis 6) suggest that higher levels of parents' and teenagers' perceptions of open family communication, parent-teenager sexual communication, and comfort with sexual communication are associated with lower levels of teenagers' attitudes and subjective norms toward having sex in the next 3 months, and higher levels of self-efficacy about avoiding risky sexual behavior. Other researchers have reported positive relations between parentteenager communication and teenagers' attitudes, subjective norms and self-efficacy toward risky sexual behavior(9,16,18-21). However, only one study reported findings specifically for Latino teenagers(21).

Paths $m, n$, and $o$ (Hypothesis 7) suggest that lower levels of teenagers' attitudes and subjective norms toward having sex in the next 3 months and higher levels of self-efficacy about avoiding risky sexual behavior are associated with lower levels of teenagers' intentions to have sex in the next 3 months. Previous studies have shown relationships between teenagers' attitudes, subjective norms, and self-efficacy about avoiding risky sexual behavior and teenagers' sexual risk behaviors ${ }^{(22-25)}$.

The present study assessed individually the components of the PETPB model with a sample of Latino teenagers and parents in the United States, and analyzed both teenagers' and parents' reports of parent-teenager general communication, sexual communication, and comfort with sexual communication.

\section{Methods}

A cross-sectional correlational design was used based on a secondary analysis of baseline data collected as part of a randomized control trial designed to test the efficacy of a parent computer-based intervention designed to increase Latino parent-teenager sexual communication ${ }^{(26)}$. The participants in this study were recruited from Southwest Detroit, in a Latino community area with the largest concentration of Latinos in the state of Michigan. Recruitment was through fliers, advertisement in a bilingual newspaper, and via personal contact and presentations made at schools and community-based programs, such as English and health promotion courses. This study analyzed the preassessment questionnaires that were completed by the sample of 130 Latino parents and their 130 teenagers (between ages of 12 - 18). Institutional Review Board approval for the secondary analysis was obtained from the University of Alabama at Birmingham.

\section{Measures}

The measures used in this study were previously translated, back-translated, pilot tested, modified in Spanish, and were used with Mexican parents in previous 
studies $^{(14,26)}$. Table 1 shows the Cronbach alpha internal consistency reliability for the study measures, which ranged from 0.71 to 0.95 . Items were measured with 5-point Likert-type scales with higher scores indicating greater perceptions of attitudes, subjective norms, and self-efficacy toward sexual communication with teenager and higher levels of familism, knowledge, communication and comfort with sexual communication. The validity of all the instruments was previously reported(26-27).

The Latino acculturation scale used preferred language as a proxy measure of acculturation and included parents' and teenagers' reports of their preferences for using English or Spanish for speaking, reading, and the preferred language used in the home. Higher scores indicated higher Latino acculturation and lower scores indicated Anglo acculturation. Familism was measured with an adapted version of the Attitudinal Familism Scale(28) which included four interrelated dimensions of familism: (a) familial obligations (six items); (b) perceived support from the family (one item); (c) family as referents (seven items). The same scale was used with both parents and teenagers. Pregnancy/ STD/AIDS knowledge was measured with a test of six questions that assessed teenagers' and parents' knowledge about pregnancy, STD and HIV transmission. Test-retest reliability of Pregnancy/STD/AIDS knowledge was calculated and showed a positive and significant Pearson correlation coefficient for the parents $(r=0.753$, $p=0.000)$ and teenagers $(r=0.765, p=0.000)$.

The scale to measure parents' attitudes toward sexual communication with their teenager assessed parents' feelings about talking with their teenager about sex, contraceptive methods, and condom use. The scale to measure parents' subjective norms toward sexual communication with their teenager assessed parents' perceptions of what people considered important and whether the parents would approve or disapprove of their talking about sex, contraceptive methods, and condoms with their son/daughter. The scale to measure parents' perceived self-efficacy toward sexual communication with their teenager assessed parents' perception of whether he/she had sufficient resources, skills, and confidence to communicate with his/her teenager about sex, contraceptive methods, and condom use. The scale measuring parents' intentions to talk about sexual behavior assessed the parent's perceived subjective probability that he/she would talk with their daughter or son in the next 3 months about sex, contraceptive methods, and condoms.

Open family communication was measured with the Parent-Teenager Communication Openness Subscale(29) that assessed openness of general communication between parent and teenager. Parent-teenager sexual communication was measured by the Parent-Teen Sexual Risk Communication Scale ${ }^{(19)}$ that assessed the amount of information the parents had shared with the teenager during the past 3 months about parents' feelings about the teenagers' sexual behavior, contraception, STDs, HIVs, protection from STDs and HIV, condoms, postponing or not having sex, peer pressure and sexual pressure from peers and dating partners, and how to resist pressure from peers and dating partners. The Comfort with Sexual Communication Scale ${ }^{(20)}$ included eight questions that assessed the level of comfort in discussing various sexual topics (e.g., How comfortable do you feel when you talk to your parents/teenagers about sexual themes, behaving sexually, how to prevent pregnancy, STDs, HIV/AIDS, protection from STDs and HIVs, condoms specifically, sexual pressure from peers and dating partners?).

The scale measuring teenagers' attitudes regarding sexual communication with parents assessed the degree to which the teenagers have a positive or negative evaluation or appraisal of parent-teenager sexual communication(25). The scale measuring teenagers' subjective norms regarding sexual communication with parents assessed the teenagers' perception of whether people considered important to them would approve or disapprove their talking with parents about sex, contraceptive methods, and condom use ${ }^{(14)}$. The scale measuring teenagers' self-efficacy about avoiding risky sexual behavior measured the teenagers' perceptions of whether they had sufficient resources, skills, and confidence to exert self-control and not have sex, even if their partner wanted to; or to use condoms, even if the partner didn't want to; or to use some method of contraception, even if the partner objected(25).

Teenagers' attitudes toward having sex in the next 3 months was measured with one single question: "How do you feel about having sex in the next 3 months?" Answers ranged from 1 (very bad idea) to 5 (very good idea). Teenagers' subjective norms toward having sex in the next 3 months was measured with one question "Would most people who are important to you approve or disapprove of your having sex in the next 3 months?" Answers ranged from 1 (they would strongly disapprove) to 5 (they would strongly approve). Teenagers' intentions to have sex in the next 3 months were measured with one question "How likely is it that you will decide to have sex in the next 3 months?". Responses ranged from 1 (very unlikely) to 5 (very likely). 
Table 1 - Distribution of Internal Reliability for Teenagers' and Parents' Scales. Detroit, MI, USA, 2009-2010

\begin{tabular}{|c|c|c|}
\hline \multirow{2}{*}{ Instruments (and number of items) } & Parent Scales & Teenager Scales \\
\hline & Cronbach's $\alpha$ & Cronbach's $\alpha$ \\
\hline Latino acculturation scale ( 3 items) & 0.95 & 0.78 \\
\hline Familism scale (14 items) & 0.79 & 0.71 \\
\hline Open family communication scale (10 items) & 0.73 & 0.88 \\
\hline Parent-teenager sexual communication scale (9 items) & 0.95 & 0.93 \\
\hline Comfort with sexual communication scale (8 items) & 0.94 & 0.95 \\
\hline Teenagers' attitudes regarding sexual communication with parents scale ( 3 items) & - & 0.85 \\
\hline Teenagers' subjective norms regarding sexual communication with parents scale ( 3 items) & - & 0.87 \\
\hline Teenagers' self-efficacy about avoiding risky sexual behavior scale (4 items) & - & 0.72 \\
\hline Parents' attitudes toward sexual communication with their teenager scale (3 items) & 0.72 & - \\
\hline Parents' subjective norms toward sexual communication with their teenager scale ( 3 items) & 0.86 & - \\
\hline Parents' perceived self-efficacy toward sexual communication with their teenager scale (5 items) & 0.92 & - \\
\hline Parents' intentions to talk about sexual behavior scale ( 3 items) & 0.89 & - \\
\hline
\end{tabular}

\section{Data analysis}

Data analysis was conducted using IBM SPSS Version 20 and IBM SPSS Amos 20. A level of significance of .05 was considered statistically significant. For each of the seven research hypotheses, the data were analyzed in two stages: an exploratory stage (using simple and multiple linear regression to examine the relationships between independent and dependent variables specific to each hypothesis), and a confirmatory stage (using path analysis techniques to test the extent to which the relationships identified in the exploratory stage supported the proposed model). Goodness of fit for the path models were evaluated by the comparative fit index (CFI), and the root mean square error of approximation (RMSEA). CFI values of .95 or greater and RMSEA values of .06 or less are indicative of good model fit, and RMSEA values larger than .10 are indicative of poor-fitting models ${ }^{(30)}$. Effect sizes and Cohen's ${ }^{(31)}$ guidelines were used to describe the magnitude of the relationship between the variables. Power analysis for multiple regression models showed adequate power to detect medium effect sizes ( $80 \%$ power to detect an $\mathrm{R}^{2}$ ranging from 0.06 to 0.08 , depending on the hypothesis); and for the path models, using a test of RMSEA, the sample size provided adequate power ( $80 \%$ ) to test for goodness of fit in simple models (with higher degrees of freedom), but provided only moderate power (ranging from 58\% to $69 \%$ ) for models with greater complexity (and therefore less degrees of freedom).

\section{Findings}

\section{Sample Characteristics}

The parents were mostly female $(n=107,82.9 \%)$, with a mean age of 39.26 (SD of 7.104 ), and $54.2 \%$
(65) had not completed high school. A total of $69.9 \%$ (86) were married and $81.5 \%$ (106) reported that they were of Mexican origin. The length of time they had lived in the United States averaged 19.37 years (SD of 12.4). A total of $70(53.8 \%)$ of the teenagers in the sample were female, and their mean age was 14 years $(S D=1.489)$. Only one teenager reported that he/she was not currently enrolled at school.

Hypothesis 1: Higher parental levels of Latino acculturation, familism values, and knowledge about pregnancy/STD/AIDS are associated with more positive parental attitudes, greater subjective norms, and higher levels of self-efficacy toward sexual communication with their teenagers.

In the exploratory stage, only two predictors, parent familism (Std. $\beta=0.218, p=0.017$ ) and parent knowledge about pregnancy/STD/AIDS (Std. $\beta=0.254, \quad p=0.009$ ), were significantly associated with the outcome of parents' attitudes toward sexual communication with their teenagers. Both predictors had medium effect on the outcome of parents' attitudes toward sexual communication with their teenagers. In the second regression model only the predictors of parent Latino acculturation (Std. $\beta=0.186, p=0.042$ ) and parent familism (Std. $\beta=0.256, p=0.005$ ) were significantly associated with the outcome of parents' subjective norms toward sexual communication with their teenagers. Parent Latino acculturation (Std. $\beta=-0.28, p=0.002$ ) was negatively associated with parents' self-efficacy toward sexual communication with their teenagers. Parental knowledge about pregnancy/ STD/AIDS (Std. $\beta=0.232, p=0.012$ ) was positively associated with higher levels of parents' self-efficacy toward sexual communication with their teenagers. Both Latino acculturation and parental knowledge had a medium effect on the outcome of parents' self-efficacy 
toward sexual communication with their teenagers. The confirmatory stage for the first hypothesis supported the findings from the multiple regression analyses. The path analysis suggested a good fit of the model to the data for the hypothesis one, with two of the three indices providing support for the null hypothesis that the model fit the data well (Figure 2).

Hypothesis 2: Higher levels of parental attitudes, subjective norms, and perceived self-efficacy toward sexual communication with their teenager are associated with higher levels of parental intentions to talk about sexual behavior with their teenagers.

In the exploratory stage, only the predictor of parents' subjective norms toward sexual communication with their teenager was significantly associated with the outcome of parents' intentions to talk about sexual behavior with their teenagers (Std. $\beta=0.347, p=0.000$ ). This predictor had a medium effect on the outcome. The confirmatory stage supported the findings from the multiple regression analysis and showed a good fit model for two of the evaluation parameters for goodness of fit (see Figure 2).

Hypothesis 3: Higher levels of parental intentions to talk about sexual behavior with their teenagers are associated with greater open family communication, parent-teenager sexual communication, and parentteenager comfort with sexual communication, reported by both parents and teenagers.

In the exploratory stage, the predictor of parents' intentions to talk about sexual behavior with their teenager was significantly associated with three outcomes: parent-teenager sexual communication perceived by parent (Std. $\beta=0.217, p=0.013$ ), open family communication perceived by teenager (Std. $\beta=0.193, p=0.028$ ), and comfort with sexual communication perceived by teenager (Std. $\beta=0.214$, $p=0.015)$. However, the results of the path analysis in the confirmatory stage suggested that the model did not fit the data (Figure 2). Figure 2 shows the model fit statistics presented in shaded boxes.

Hypothesis 4: Higher teenager levels of Latino acculturation, familism, and knowledge about pregnancy/STD/AIDS are associated with higher levels of their attitudes and subjective norms about sexual communication with parents.

In the exploratory stage, only the predictor of teenager knowledge about pregnancy/STD/AIDS was significantly associated with higher levels of teenagers' attitudes (Std. $\beta=0.223, p=0.012$ ) and subjective norms about sexual communication with parents (Std. $\beta=0.208, p=0.020)$. The results of the path analyses in the confirmatory stage suggested that the model was a good fit to the data (see Figure 3 ). Figure 3 shows the model fit statistics presented in shaded boxes.

Hypothesis 5: Higher levels of teenagers' attitudes and subjective norms regarding sexual communication with parents are associated with higher levels of parents' and teenagers' perceptions of open family communication, parent-teenager sexual communication, and comfort with sexual communication.

In the exploratory stage, only the predictor of teenagers' attitudes regarding sexual communication with parents was significantly associated with higher levels of open family communication (Std. $\beta=0.637, p=0.000$ ), parent-teenager sexual communication (Std. $\beta=0.399$, $\mathrm{p}=0.000$ ), and comfort with sexual communication (Std. $\beta=0.486, p=0.000$ ) perceived by the teenagers. In the confirmatory stage, the path model suggested that the model did not have a good fit to the data (see Figure 3).

Hypothesis 6: Higher levels of parent and teenager perceptions of open family communication, parentteenager sexual communication, and comfort with sexual communication are associated with lower levels of teenagers' attitudes and subjective norms toward having sex in the next 3 months, and higher levels of self-efficacy about avoiding risky sexual behavior.

Only the predictor of teenagers' subjective norms toward having sex in the next 3 months was significantly associated with the outcome of comfort with sexual communication perceived by teenager (Std. $\beta=0.357, p=0.014$ ). In other words, teenagers who perceived approval from significant others for them to have sex in the next 3 months reported higher comfort with sexual communication with parents. Issues with multicollinearity were evidenced between the predictors of parent-teenager sexual communication and comfort with sexual communication perceived by parents, which means that these two variables were redundant. In the confirmatory stage, the path model suggested that the model did not provide good fit to the data (see Figure 3).

Hypothesis 7: Lower levels of teenagers' attitudes and subjective norms toward having sex in the next 3 months and higher levels of self-efficacy about avoiding risky sexual behavior are associated with lower levels of teenagers' intentions to have sex in the next 3 months.

Only the predictor of teenagers' attitudes toward having sex in the next 3 months was significantly associated with the outcome of teenagers' intentions to have sex in the next 3 months (Std. $\beta=0.721$, $p=0.000)$. These results suggest that teenager 
attitudes toward having sex in the next 3 months is an important variable to be considered when designing future programs to decrease teenagers' intentions to have sex. In the confirmatory stage, the path model suggested that the model did not provide good fit to the data (Figure 3).

\section{External influences on Parent:}

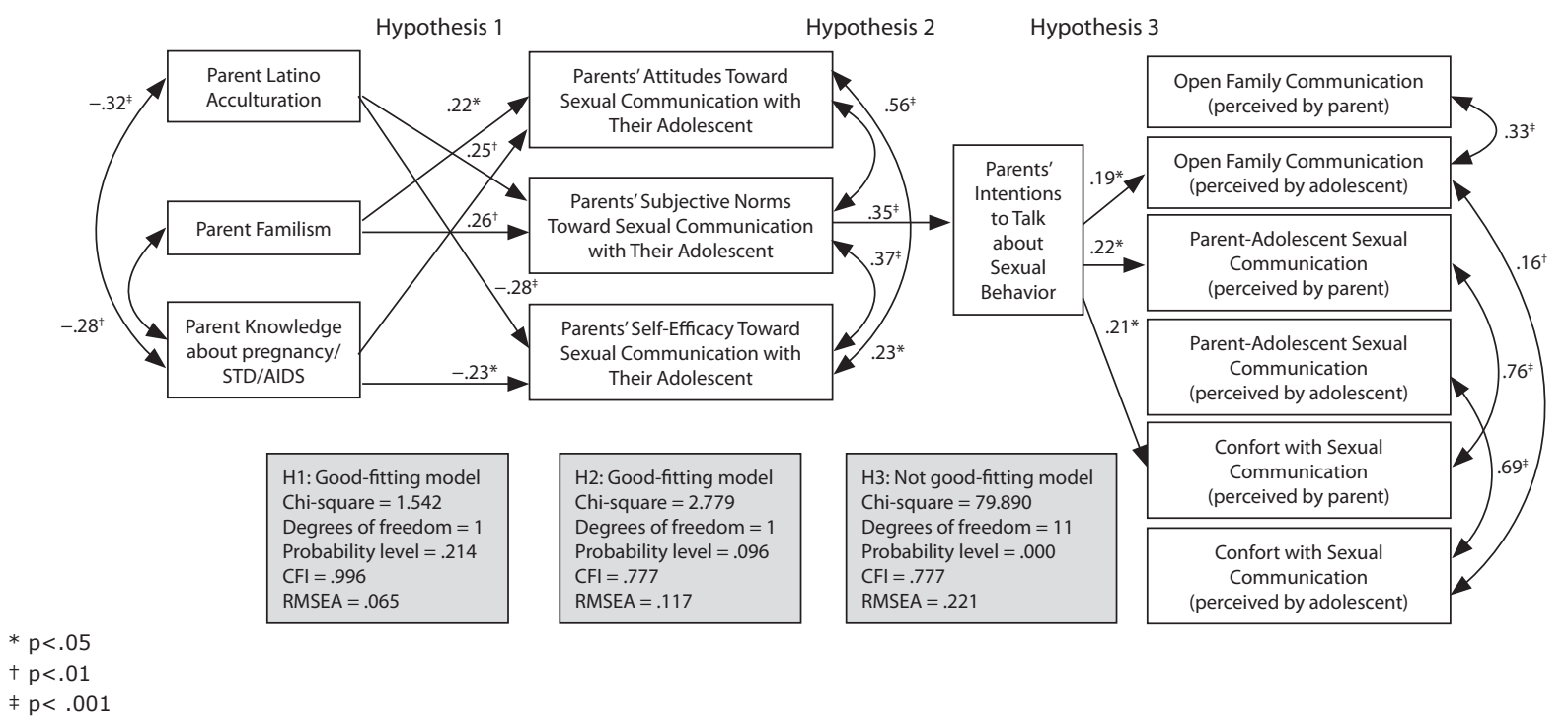

Figure 2 - Summary of Path Model for Hypotheses 1-3 with the standardized regression coefficients. Detroit, MI, USA 2009-2010

External influences on Adolescent:

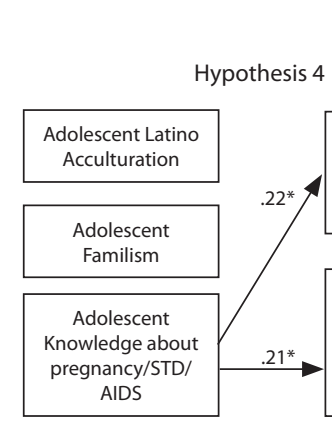

\begin{tabular}{|l|}
\hline H4: Good-fitting model \\
Chi-square $=2.346$ \\
Degrees of freedom $=3$ \\
Probability level $=.504$ \\
CFI $=1.0$ \\
RMSEA $=.000$ \\
\hline
\end{tabular}

$* \mathrm{p}<.05$

$+p<.01$

$\neq \mathrm{p}<.001$

Figure 3 - Summary of Path Model for Hypotheses 4-7 with the standardized regression coefficients. Detroit, MI, USA 2009-2010

\section{Discussion}

The PETPB model posits that external variables may influence parents' behavioral beliefs, normative beliefs, control beliefs, and intentions, which then influence teenager behavioral, normative and control beliefs, and thus influence teenagers' risky sexual behavior(4). Because of the small sample size (130 parents and 130 
teenagers), it was not feasible to conduct a statistical analysis to test the full model at one time. Instead, seven separate analyses were conducted to test hypotheses that were developed based on findings from previous research and on the relationships predicted by the model. Figures 2 and 3 illustrate the relationships in the model that were supported by the findings from this study.

The findings from this study provide partial support for each of the hypotheses that were derived from the conceptual model that guided the study, as noted in Figure 1. Although, parent-teenager sexual communication may influence teenager risky sexual behavior, there is limited evidence about how parental sexual communication may influence the teenagers' attitudes, subjective norms and self-efficacy toward risky sexual behavior.

The results of the first hypothesis suggest that parent Latino acculturation, parent familism, and parent knowledge about pregnancy/STD/AIDS are significantly related to parents' attitudes, subjective norms, and self-efficacy toward sexual communication with their teenager. Unexpectedly, parent Latino acculturation was negatively associated with parents' self-efficacy toward sexual communication with their teenagers. One explanation for this may be that the Latino acculturation variable was measured only with questions related to language of preference. Using a more comprehensive measure of acculturation may have yielded different results. The mean for the Latino acculturation scale was higher for parents (mean=4.11) than teenagers (mean=2.83). The different language preferences of parents and teenagers may have created a barrier for parent-teenager sexual communication, and may explain the negative association between parental Latino acculturation and self-efficacy towards sexual communication. No previous studies were identified that examined the relationship between these specific variables, although studies have identified positive relationships between actual parent-teenager communication and parental acculturation (sample of Latino teenagers, males and females, mean age of 13.6 years, living in the U.S.)(5), sexual knowledge (sample of Mexican teenagers, males and females, ages 14-17 years, living in Mexico $)^{(7)}$, and parental familism and selfefficacy toward parent-teenager sexual communication (sample of Mexican teenagers, males and females, ages 14-17 years, living in Mexico)(6).
The second hypothesis was partially supported. Parental subjective norms toward sexual communication with their teenagers are positively related to their intentions to talk with their teenagers about sex. There were no signficant relations between parental attitudes and self-eficacy towards sexual communication and intentions to talk about sexual behavior. One explanation for this finding may be the homogeneity of responses on parental attitudes and parental intentions towards sexual communication. Most of the parents (87\%) responded that sexual communication with their teenagers was "a good idea" or "a very good idea" and 84\% of the parents were "likely" or "very likely" to talk about sex with their teenager. No previous studies were identified that specifically measured relationships examined in the second hypothesis between parents' intentions to talk about sexual behavior and parents' attitudes, subjective norms, and self-efficacy toward sexual communication with their teenagers. However, previous studies have demonstrated positive relationships between actual parent-teenager sexual communication and parents' attitudes, subjective norms, and self-efficacy toward sexual communication ${ }^{(6,11-14)}$ (a sample of Mexican parents who had teenagers between 14 and 17 years of age, male and female, living in Mexico(6); predominately African-American mothers and teenagers, ages 11-14, male and female, living in the U.S. (12); predominately Latino mothers and teenagers, mean age of 13 , male and female, living in the U.S.(13); Mexican parents, predominately mothers, living in Mexico ${ }^{(14)}$ ).

The findings did not support the model proposed by the third hypothesis that there would be positive relations between parental intentions to talk about sexual behavior and communication variables reported by both parents and teenagers. However, findings from regression analyses suggested that three variables (open family communication perceived by the teenager, parent-teenager sexual communication and comfort with sexual communication perceived by the parent) were related to parental intentions to talk about sexual behavior. One explanation for the failure to support the full model may be the interrelationships among the multiple communication variables. No previous studies were identified that specifically examined the relations tested in the third hypothesis.

Only one of three relations that were predicted in the fourth hypothesis was supported by the findings. 
Teenager sexual knowledge (but not Latino acculturation or familism) was related to teenagers' attitudes and subjective norms regarding sexual communication with parents. Although no previous studies were identified examining these specific relationships, two studies were identified that reported positive relations between teenager sexual knowledge and perceptions of actual parent-teenager sexual communication ${ }^{(16,32)}$, (sample of teenager African American males, aged 18-22, living in the U.S.(16); predominately white teenagers, males and females, aged 14-18, living in the U.S. ${ }^{(32)}$ ), consistent with the findings reported in the present study.

Findings related to the fifth hypothesis suggested that teenager attitudes towards sexual communication with parents (but not their subjective norms) were related to teenager (but not to parent) perceptions of open family communication, parent-teenager sexual communication, and comfort with sexual communication. However, the data did not support the path model for this hypothesis. These findings contradicted findings from one study (that did not include Latinos, had a sample of teenagers with mean age of 16 years, male and female, of mixed race, living in the Netherlands) which reported a positive association between teenagers' beliefs and subjective norms toward parent-teenager sexual communication and teenagers' reports of parentteenager sexual communication(17).

The sixth hypothesis did not support the path model that predicted relationships between different parent-teenager communication variables and teenagers' attitudes, subjective norms, and self-efficacy about avoiding risky sexual behavior. Teenagers' perceived comfort with parent-teenager communication (but not parent or teenager perceptions of open family communication or parent-teenager sexual communication) was related to teenagers' subjective norms toward having sex in the next 3 months. Only one other study was found that examined these relationships in Latino families (Latino mothers and teenagers, aged 12-15, male and female, predominately from Mexico, living in the U.S.), and findings from this study suggested positive relationships between open family communication and teenager attitudes toward premarital sex ${ }^{(21)}$.

The seventh hypothesis was also only partially supported by the data. Teenagers' attitudes (but not subjective norms or self-efficacy) toward having sex in the next 3 months were positively associated with their intentions to have sex in the next 3 months. This result might be explained by the fact that only 11 teenagers reported intentions to have sex in the next 3 months, and thus there may not have been sufficient variability to identify significant relations. This finding was consistent with findings reported previously ${ }^{(22,25)}$ that favorable attitudes (but not subjective norms or selfefficacy) toward engaging in sexual intercourse were related to sexual activity (sample of African-American teenager females, age $12-18^{(22)}$; Latino teenagers, male and female, age 12-18, living in the U.S. $\left.{ }^{(25)}\right)$.

There were some limitations to the current study that should be addressed in order to provide directions for future research. First, the secondary data analyses were derived from a randomized controlled trial - preintervention survey, thus limiting the ability to identify longitudinal predictive relationships. Second, the measure used to assess acculturation focused on the respondents' preferred language, and did not include other components of acculturation such as values and group affiliation. A third limitation was the use of a convenience sample included Latinos who were primarily of Mexican origin (81.5\%) who had lived in the United States for an average of 19.37 years. In addition, 83\% of the parents in the sample were mothers. Further, a covariate adjusted analysis that could have included factors possibly affecting the relationships examined in the study (e.g., length of time in the U.S., education, and family income) was not conducted due to sample size limitations. A fourth limitation was that the study data were based on self-report measures completed by young teenagers and parents. Accuracy of the reports could be affected by fear of exposing oneself on sensitive topics such as intention to engage in sexual intercourse, even though the questionnaires were administrated through computers in a private room. A fifth limitation was that very few teenagers in the sample reported intentions to have sex in the next 3 months, resulting in limited variability in the primary dependent variable and thus limiting the power to detect predictive relationships. A final limitation of this study was that data for males and females were not analyzed separately.

There are a number of implications of the findings for future research. Future studies should be longitudinal to examine how cultural variables and family factors are related to teenagers' sexual intentions over time. 
Future studies should also use different measures of acculturation that are more multi-dimensional and which include values of gender role. Findings from previous studies suggest that there may be different relations between parent-teenager communication and teenager sexual behaviors for the two genders ${ }^{(18)}$, and thus future studies should test the PETPB model separately for male and female teenagers. Finally, future studies should include a larger sample of Latino mothers and fathers from different countries and with more variation in levels of acculturation in order to generalize the findings to the diverse Latino immigrant population living in the United States, and allow examination of covariate effects.

Study findings also have important implications in practice. Health care providers caring for Latino teenagers should assess teenager levels of acculturation, sexual knowledge, attitudes, norms, and intentions to have sex as well as parent-teenager communication variables in order to develop tailored interventions to reduce risky sexual behavior.

\section{Conclusion}

The results of this study provide important information to guide future research that can inform development of interventions to prevent risky teenager sexual behaviors among Latinos. The findings suggest that the PETBP model provides a useful framework for understanding the complex relations between selected parental, teenager and cultural variables and Latino teenagers' intentions to engage in sexual behavior. Future research should also explore use of this model in different countries and with different cultural groups.

\section{References}

1. Pew Research Center. Median age for Hispanics is lower than median age for total U.S. population. 2012.[acesso 11 jan 2015]. Disponível em: http://www.pewresearch. org/daily-number/median-age-for-hispanics-is-lowerthan-median-age-for-total-u-s-population/

2. Centers for Disease Control and Prevention. Youth Risk Behavior Surveillance - United States 2013. [acesso 19 jan 2015]. MMWR Surveillance Summaries. [Internet]. 2014;63(4):1-172. Disponível em: http://www.cdc.gov/ healthyyouth/yrbs/
3. Centers for Disease Control and Prevention. Health Disparities in HIV/AIDS, Viral Hepatitis, STDs, and TB. 2014 [Internet]. [acesso 22 dez 2014]. Disponível em: http://www.cdc.gov/nchhstp/healthdisparities/Hispanics. html\#HIV

4. Coatsworth JD, Pantin H, Szapocznik J. Familias Unidas: a family-centered ecodevelopmental intervention to reduce risk for problem behavior among Hispanic adolescents. Clin Child Fam Psychol Rev. 2002;5(2):113-32. doi: 10.1023/A:1015420503275

5. Ortega J. Applying Ecodevelopmental Theory and the Theory of Reasoned Action to understand HIV risk behaviors among Hispanic adolescents [Doctoral dissertation]. Miami (FI): University of Miami; 2010. 140 p.

6. Benavides RA. An Interaction Model of Parents' and Adolescents' Influences on Mexican Adolescents' Intentions for Contraception and Condom Use [Doctoral dissertation]. Austin, Texas: University of Texas at Austin; 2007. 206p.

7. Gallegos EC, Villarruel AM, Gómez MV, Onofre DJ, Zhou Y. Research brief: sexual communication and knowledge among Mexican parents and their adolescent children. J Assoc Nurses AIDS Care. 2007;18(2):28-34. doi: 10.1016/j.jana.2007.01.007

8. Lugo Steidel A, Contreras J. A new familism scale for use with Latino populations. Hispanic J Behav Sci. 2003;25(3):312-30. doi: 10.1177/0739986303256912

9. Hutchinson MK, Jemmott III JB, Sweet Jemmott L, Braverman P, Fong GT. The role of mother-daughter sexual risk communication in reducing sexual risk behaviors among urban adolescent females: A prospective study. J Adolesc Health. 2003;33(2):98107. doi: $10.1016 /$ S1054-139X(03)00183-6

10. Hutchinson MK, Wood EB. Reconceptualizing adolescent sexual risk in a Parent Based Expansion of the Theory of Planned Behavior. J Nurs Scholarsh. 2007;39(2):141-6. doi: 10.1111/j.15475069.2007.00159.x

11. Benavides RA. Comunicacion y creencias sexuales de padres de adolescentes [Master thesis]. Monterrey, México: Universidad Autónoma de Nuevo León; 2003. $30 \mathrm{p}$.

12. DiIorio C, Resnicow K, Dudley WN, Thomas S, Wang DT, Van Marter DF, et al. Social cognitive factors associated with mother-adolescent communication about sex. J Health Commun. 2000;5(1):41-51. doi: $10.1080 / 108107300126740$ 
13. Guilamo-Ramos V, Jaccard J, Dittus P, Collins S. Parent-adolescent communication about sexual intercourse: an analysis of maternal reluctance to communicate. Health Psychol. 2008; 27(6):760-9. doi: $10.1037 / \mathrm{a} 0013833$

14. Villarruel $A M$, Cherry $C L$, Cabriales EG, Ronis DL, Zhou Y. A parent-adolescent intervention to increase sexual risk communication: Results of a randomized controlled trial. AIDS Educ Prevent: official publication of the International Society for AIDS Education. 2008;20(5):371-83. doi: 10.1521/aeap.2008.20.5.371. 15. Chung PJ, Travis Jr R, Kilpatrick SD, Elliott MN, Lui C, Khandwala SB, et al. Acculturation and parentadolescent communication about sex in FilipinoAmerican families: a community-based participatory research study. J Adolesc Health. 2007;40(6):543-50. doi: 10.1016/j.jadohealth.2007.01.004

16. Harris $A L$, Sutherland $M A$, Hutchinson $M K$. Parental Influences of Sexual Risk Among Urban African American Adolescent Males. J Nurs Scholarsh. 2013;45(2):1-10.

17. Schouten BC, Van den Putte B, Pasmans $M$, Meeuwesen L. Parent-adolescent communication about sexuality: The role of adolescents' beliefs, subjective norm and perceived behavioral control. Patient Educ Counseling. 2007;66(1):75-83. doi: 10.106/j. pec. 2006.10.010

18. Hutchinson MK, Montgomery AJ. Parent communication and sexual risk among African Americans. Western J Nurs Res. 2007;29(6):691-707.

19. Hutchinson MK, Cooney TM. Patterns of parent-teen sexual risk communication: Implications for intervention. Fam Rel. 1998;47(2):185-94.

20. DiIorio C, Kelley $M$, Hockenberry-Eaton $M$. Communication about sexual issues: Mothers, fathers, and friends. J Adolesc Health. 1999;24(3):181-9. doi: 10.1016/S1054-139X(98)00115-3

21. Romo LF, Lefkowitz ES, Sigman M, Au TK. A longitudinal study of maternal messages about dating and sexuality and their influence on Latino adolescents.

J Adolesc Health. 2002; 31(1):59-69. doi: 10.1016/ s1054-139x(01)00402-5

22. Childs G, Moneyham L, Felton G. Correlates of sexual abstinence and sexual activity of low-income African American adolescent females. J Assoc Nurses AIDS Care. 2008;19(6):432-42. doi: 10.1016/j.jana.2008.04.013
23. Koniak-Griffin D, Stein JA. Predictors of sexual risk behaviors among adolescent mothers in a human immunodeficiency virus prevention program. J Adolesc Health. 2006;38:297.e1-e11. doi: 10.1016/j. jadohealth.2004.12.008

24. Prado G, Schwartz S, Maldonado-Molina M, Huang S, Pantin H, Lopez $B$, et al. Ecodevelopmental $x$ Intrapersonal risk: Substance use and sexual behavior in Hispanic adolescents. Health Educ Behavr. 2009; 36(1):45-61. doi: 10.1177/1090198107311278

25. Villarruel AM, Jemmott III JB, Jemmott LS, Ronis DL. Predictors of sexual intercourse and condom use intentions among Spanish-dominant Latino youth: a test of the planned behavior theory. Nurs Res. 2004; 53(3):172-81. doi:10.1097/00006199-20040500000004

26. Villarruel AM, Loveland Cherry CJ, Ronis DL. Testing the efficacy of a computer based parent adolescent sexual communication intervention for Latino parents. Fam Rel. 2010; 59(5):533-43. doi: 10.1111/j.17413729.2010.00621.x

27. Gaioso VP. Relationships between Latino parental, adolescent and cultural variables on adolescents' attitudes, norms, self-efficacy, and sexual intentions [Dissertation]. Birmingham (AL): University of Alabama at Birmingham School of Nursing; 2013. 258 p. [acesso 19 dez 2014]. Disponível em: http://search. proquest.com/docview/1434877976/ abstract/14199FA13B736AAD6FD/1 ?accountid $=8240$

28. Sabogal F, Marín G, Otero-Sabogal R, Marín B, Perez-Stable E. Hispanic familism and acculturation: What changes and what doesn't? Hispanic J Behav Sci. 1987;9(4):397-412. doi: 10.1177/07399863870094003

29. Barnes H, Olson DH. Parent-adolescent communication scale. In: Olson DH, McCubbin HI, Barnes $H$, Larsen A, Muxen M, Wilson M. Family inventories: Inventories used in a national survey of families across the family life cycle. St. Paul (MN): Family Social Science, University of Minnesota Press; 1982. p. 33-48.

30. Tabachnick B, Fidell L. Using multivariate statistics. New York: Allyn and Rose; 2007.

31. Cohen J. Statistical power analysis for the behavioral sciences. Hillsdale, N.J.: Lawrence Erlbaum; 1988. 
32. Somers $\mathrm{CL}$, Paulson SE. Students' perceptions of parent-adolescent closeness and communication about sexuality: Relations with sexual knowledge, attitudes, and behaviors. J Adolesc. 2000;23(5):629-44. doi:

$10.1006 /$ jado. 2000.0349 